OPEN ACCESS

Edited by:

Margaret Ip,

The Chinese University of Hong Kong, Hong Kong

Reviewed by: Aleksandra Barac, University of Belgrade,

Serbia

Veranja Liyanapathirana, University of Peradeniya,

Sri Lanka

*Correspondence:

Tim G. J. de Mei

t.demeij@vumc.n!

Specialty section:

This article was submitted to Infectious Diseases - Surveillance,

Prevention and Treatment, a section of the journal

Frontiers in Medicine

Received: 22 June 2017 Accepted: 16 August 2017

Published: 30 August 2017

Citation:

de Meij TGJ, van Wijk MP, Mookhoek A and Budding AE (2017)

Ulcerative Gastritis and

Esophagitis in Two Children with

Sarcina ventriculi Infection.

Front. Med. 4:145.

doi: 10.3389/fmed.2017.00145

\section{Ulcerative Gastritis and Esophagitis in Two Children with Sarcina ventriculi Infection}

\author{
Tim G. J. de Meij ${ }^{1 *}$, Michiel P. van Wijk ${ }^{1}$, Aart Mookhoek ${ }^{2}$ and Andries E. Budding ${ }^{3}$ \\ ${ }^{1}$ Department of Paediatric Gastroenterology, VU University Medical Centre, Amsterdam, Netherlands, ${ }^{2}$ Department of \\ Pathology, VU University Medical Centre, Amsterdam, Netherlands, ${ }^{3}$ Department of Medical Microbiology and Infection \\ Control, VU University Medical Centre, Amsterdam, Netherlands
}

Sarcina ventriculi is a Gram-positive, obligate anaerobic coccus, with a characteristic morphology. Only 22 cases of human infections by this microorganism, including 7 in children, have been reported in literature so far. Affected subjects usually present with abdominal pain, nausea, vomiting, and delayed gastric emptying. However, life-threatening complications, like emphysematous gastritis and gastric perforation have also been described. Gastroparesis and gastric outlet obstruction have been considered as a potential etiologic factor. All pediatric cases described thus far presented with concomitant gastrointestinal pathology, such as Helicobacter pylori gastritis, celiac disease, infection with Giardia lamblia or Candida spp. Here, we report two children with S. ventriculi infection, in whom the diagnosis was established by typical histological findings in mucosal biopsies. The first child presented with hematemesis due to ulcerative esophagitis and gastritis, the second child with a history of esophageal stricture had ulcerative gastritis. Confirmation of $S$. ventriculi infection is feasible by molecular microbiota detection methods, since this microorganism cannot be detected by classical culture techniques. Prompt treatment with antibiotics could prevent life-threatening complications.

Keywords: esophagitis, children, microbiota, IS-pro, Sarcina ventriculi, gastritis

\section{INTRODUCTION}

Sarcina ventriculi is a Gram-positive, obligate anaerobic coccus, present in the soil, with a characteristic tetrad or octet morphology. Only 22 cases of human infections by this microorganism, including 7 children, have been described in literature to date. The majority of cases presented with abdominal pain, nausea, vomiting, and delayed gastric emptying. In a few cases, infection was associated with life-threatening complications, like emphysematous gastritis and gastric perforation. Here, we report two children with a S. ventriculi infection in whom the diagnosis could be made on typical histological findings in the mucosal biopsies. The first child presented with hematemesis due to ulcerative esophagitis and gastritis, the second child with a history of esophageal stricture had ulcerative gastritis. In both children, symptoms resolved completely following targeted antibiotics.

\section{CASE PRESENTATION}

The first patient was a 12-year old girl with a history of psychomotor retardation and refractory epilepsy based on West syndrome. Furthermore, she was diagnosed twice with an episode of 
Helicobacter pylori-associated gastritis, successfully eradicated with triple therapy on both occasions. Percutaneous endoscopic gastrostomy (PEG) was performed early in life for long-term administration of enteral nutrition. She was admitted to the hospital because of mild dehydration due to intractable vomiting since 3 days, possibly including blood. Aspiration of stomach content via the PEG tube revealed retention of a large amount of brownish fluid. On physical examination, the patient was tachycardic (110 beats/min), with normal blood pressure and oxygen saturation. Palpation of the abdomen was not painful, and no other physical signs were present, besides her pre-existent neurological impairment. Laboratory investigation showed normal hemoglobin concentration ( $9.5 \mathrm{mmol} / \mathrm{l})$, mean cellular volume $94 \mathrm{fl}$, normal platelet count, and normal infection and clotting parameters. At admission, she was already on omeprazole ( $20 \mathrm{mg} /$ day), next to phenobarbital and gabapentin as antiepileptic treatment. Differential diagnosis included Mallory-Weiss syndrome, viral gastro-enteritis, esophagitis, recurrence of $H$. pylori-associated ulcer/gastritis, and bleeding from esophageal varices. Portal hypertension was excluded by abdominal ultrasound and $H$. pylori antigen stool test was negative. Omeprazole was increased to $40 \mathrm{mg} /$ day. No hematemesis was observed during the 3 days of admission, and therefore, esophagogastroduodenoscopy was not performed. After 1 month, the patient was readmitted because of status epilepticus, possibly due to insufficient intake of antiepileptic medication linked to a gradual increase of vomiting. Convulsions were adequately treated with intravenously administered midazolam. Three days prior to this second admission, the mother of the patient had aspirated large amounts of bloody retention from the stomach, but the hemoglobin level was not decreased compared to the previous admission. One day following this second admission, the patient had an episode of hematemesis, and therefore, a esophagogastroduodenoscopy was performed under general anesthesia. A timeline displaying the course of clinical symptoms is depicted in Figure 1.

Endoscopy showed a severe, erosive esophagitis of the distal $15 \mathrm{~cm}$ of the esophagus (Figure 2). Furthermore, a multifocal hemorrhagic antral and corporal gastritis was observed (Figure 3), next to two circular antral ulcers with a diameter of $5 \mathrm{~mm}$. Notably, a large amount of stomach retention was observed, indicative for delayed gastric emptying. Histological examination of esophageal and gastric biopsies showed severe ulcerative esophagitis and gastritis, next to the presence of bacteria in the gastric and esophageal mucous surface. The bacteria were arranged in tetrad and octet groups, compatible with $S$. ventriculi (Figures 4A,B). H. pylori was not observed.

Culture of the mucosal biopsies was negative, including culture for H. pylori. Subsequently, we applied a molecular microbiota profiling technique, called IS-pro, which confirmed the presence of $S$. ventriculi. Patient was treated with ciprofloxacin and metronidazole for 10 days and the symptoms of bloody stomach retention and hematemesis resolved completely within several days. Control esophagogastroduodenoscopy performed after 6 weeks showed complete healing of the gastric and esopgaheal mucosa. In the mucosal biopies, $S$. ventriculi could no longer be detected. Duration of follow-up was 12 months, during which period no recurrence of symptoms was reported.

\section{Case 2}

The second patient was a 15 -year-old Caucasian girl with a history of severe neurological impairment and epilepsy due to intracranial hemorrhage from a cerebral arteriovenous malformation at 8 years of age. She was prescribed Clobazam and Keppra as antiepileptic agents and had a PEG tube for administration of enteral nutrition. She presented with respiratory failure due to an aspiration pneumonia, for which mechanical ventilation was required. Because of the inability to insert a nasogastric tube during admission, a single-contrast study of the esophagus was performed, which showed a mid-esophageal stricture over a length of $10 \mathrm{~cm}$. An upper endoscopy was performed which confirmed the presence of a mid-esophageal pinpoint stenosis. Endoscopy was also performed through the gastrocutaneous fistula for retrograde inspection of the distal esophagus. Here,

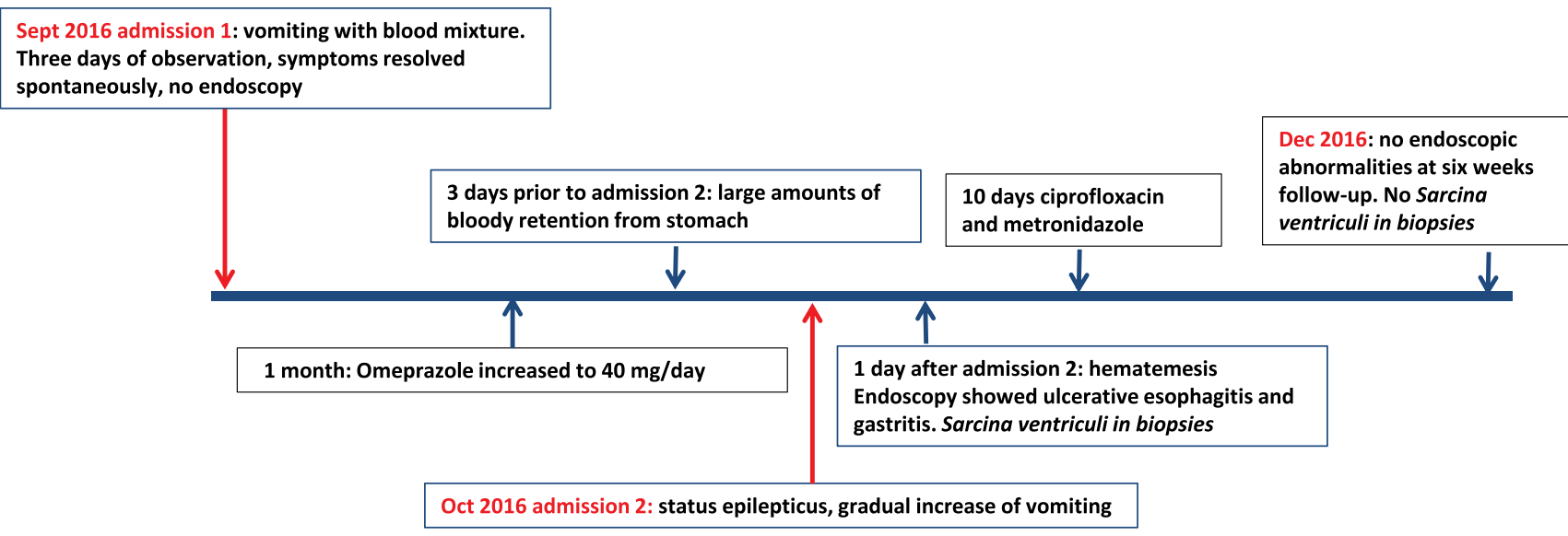

FIGURE 1 | Timeline displaying the course of clinical symptoms of case 1. 


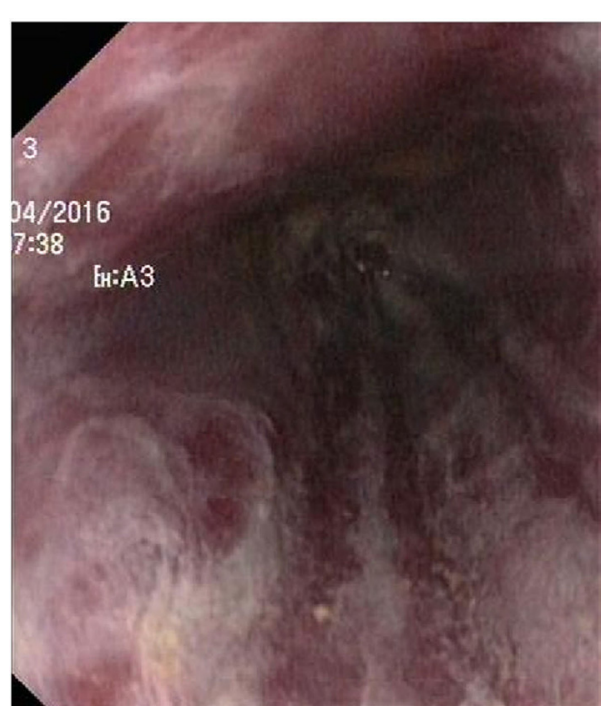

FIGURE 2 | Erosive esophagitis.

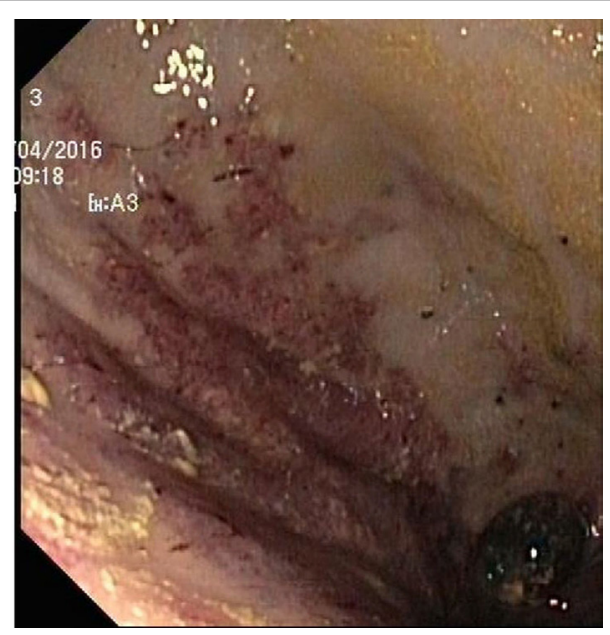

FIGURE 3 | Erosive gastritis.

we observed a diffuse erosive gastritis and a large gastric circular ulcer with a diameter of $10 \mathrm{~mm}$ at the gastro-esophageal junction with no signs of esophagitis (Figure 5). Furthermore, a significant amount of stomach retention was seen, despite $12 \mathrm{~h}$ of fasting, suggestive of delayed gastric emptying. Histological examination of gastric biopsies showed active gastritis with the presence of microorganisms arranged in tetrads, characteristic of $S$. ventriculi, which was confirmed by the IS-pro technique. H. pylori and Giardia lamblia were not detected. The patient was prescribed ciprofloxacin and metronidazole for 10 days, next to omeprazole (40 mg/day). At endoscopic follow-up 6 weeks following antibiotics, the gastritis and gastric ulcer were healed completely and $S$. ventriculi could no longer be detected from the biopsies. Endoscopic dilatation of the esophageal stricture was successfully performed using Savary-Gilliard bougies.

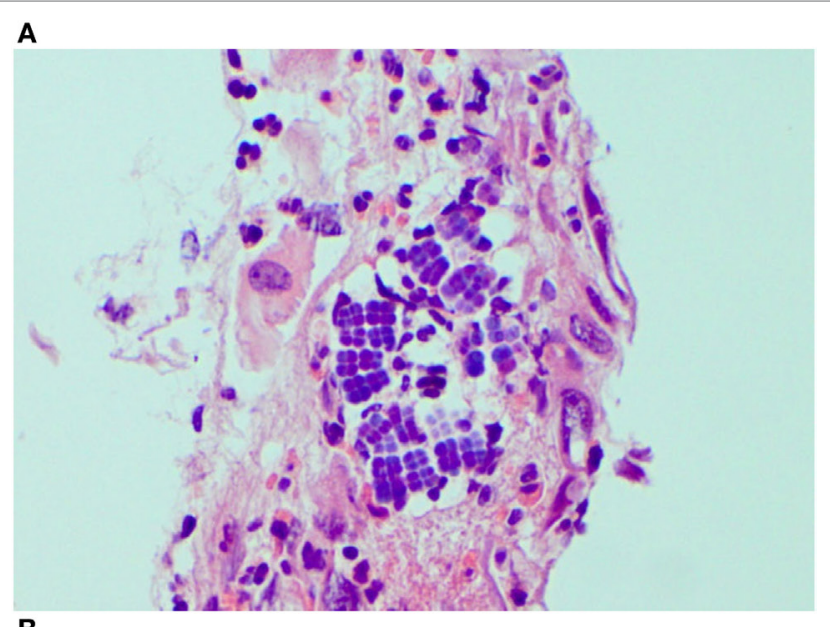

B

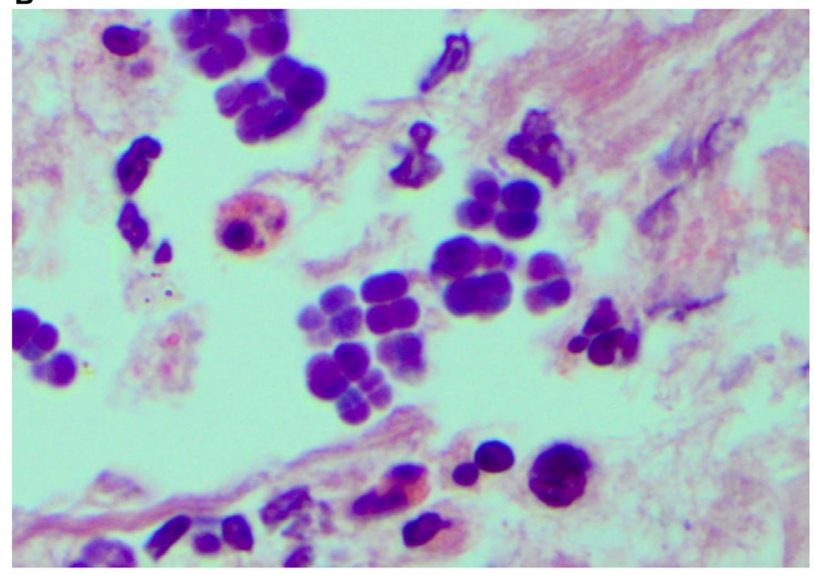

FIGURE 4 | Sarcina ventriculi in esophageal biopsies. These microorganisms appear to be arranged in tetrads [(A) 200x], but on higher magnification appear to be arranged in cubes composed of eight individual spheres [(B) 400x].

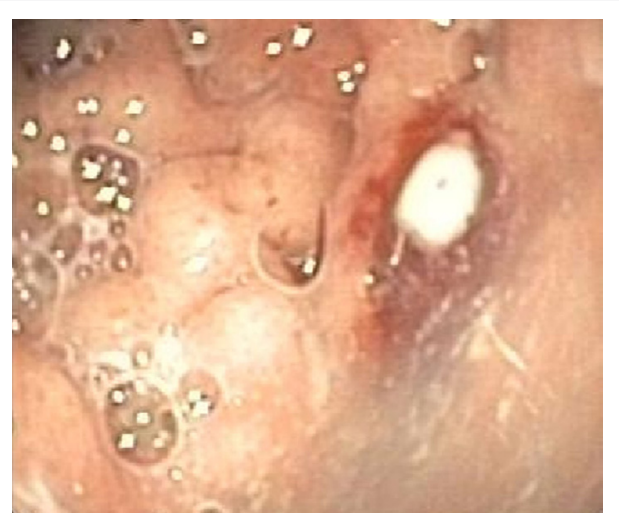

FIGURE 5 | Circular gastric ulcer.

A timeline with the course of clinical symptoms is depicted in Figure 6. During follow-up period of 8 months, no recurrence was reported. 


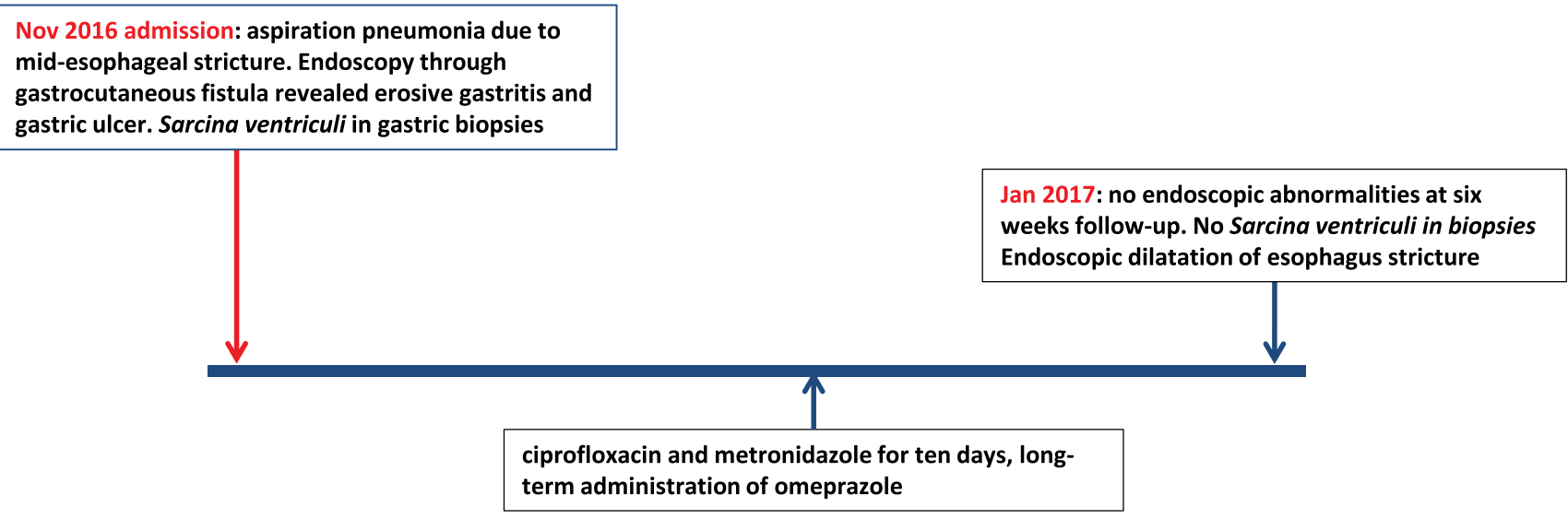

FIGURE 6 | Timeline displaying the course of clinical symptoms of case 2.

\section{DISCUSSION}

In 1842, John Goodsir was the first to describe the presence of Sarcina microorganisms in the stomach of a patient presenting with abdominal pain, bloating, and vomiting (1). The pathogenic role of $S$. ventriculi has been well established in the veterinary literature, including description of several fatal cases, called "abomasal bloat," in livestock $(2,3)$. Its pathogenicity in humans has been questioned until recent years, since only few symptomatic infections with Sarcina organisms in humans have been described, only 22 cases in literature so far (4-18), while $S$. ventriculi has also been detected in asymptomatic subjects $(19,20)$. Sites of infections described thus far include the stomach (85\%), esophagus (10\%), and duodenum (5\%) (21). Presenting symptoms in patients with S. ventriculi commonly include abdominal pain, nausea, vomiting, and, as in our case, delayed gastric emptying $(8,9)$. Severe complications like emphysematous gastritis and gastric perforation have also been reported $(4,5)$. It has been suggested that impaired gastric emptying could be a risk factor for the growth of $S$. ventriculi $(8,9)$. The majority of affected patients do not have mucosal injury on endoscopy, but increasing evidence suggests that $S$. ventriculi is associated with gastric ulcers, with an incidence of over $30 \%$ in Sarcina infections, and subsequently with an increased risk for emphysematous gastritis and gastric perforation $(7,21)$. Only seven children with symptomatic $S$. ventriculi infection have been described in literature so far $(5-7,11,12$, 22), two of them presenting with (partial) gastric necrosis. Notably, all described children had a concurrent gastrointestinal disease, including $H$. pylori gastritis $(n=2)$, infection with G. lamblia or Candida spp., history of esophageal atresia and post gastric pull through with anastomotic narrowing, and celiac disease. Our first case had no (recognized) gastrointestinal diagnosis, both cases had a PEG tube placement for administration of enteral nutrition. An association between a PEG tube and S. ventriculi infection has not been described previously. In three of seven affected children described in literature, endoscopy revealed the presence of distal esophagitis and none of them had hematemesis as presenting symptom $(7,8)$. Cultivation of $S$. ventriculi is complicated by its complex nutritional requirements, but diagnosis can usually be made based on typical morphological features (11). Diagnosis can be confirmed by means of molecular techniques like sequencing or, as in our case, by IS-pro, a eubacterial DNA-based molecular detection technique (23). IS-pro is based on identification of species-specific length polymorphisms of the interspacer region and phylum-specific sequence polymorphisms of $16 \mathrm{~S}$ rDNA. Treatment of $S$. ventriculi usually consists of proton pump inhibitors and antibiotics, with metronidazole and ciprofloxacin as most frequently prescribed agents $(3,19)$. In our first case description, the patient was already on proton pump inhibitors prior to onset of complaints, and she recovered both clinically and endoscopically following administration of ciprofloxacin and metronidazole.

The association between $S$. ventriculi infections and esophageal strictures, as described in case 2, has already been described over a century ago (22). In an intriguing experiment by Beijerinck et al. performed in 1911 under strict anaerobic conditions to prove the similarity between $S$. ventriculi isolated from garden soil and from stomach contents, the researchers used from stomach contents from patients with esophageal stenosis and suffering from $S$. ventriculi infection (22). This association has not been described in subsequent reports on human $S$. ventriculi infections. In case $2, S$. ventriculi was detected in mucosal biopsies from the stomach, but not from the esophagus. We, therefore, believe that $S$. ventriculi infection is more likely to be a consequence rather than a cause of esophageal strictures; severe stenosis may create optimal anaerobic conditions for S. ventriculi to grow.

In conclusion, $S$. ventriculi infection is a rare cause of ulcerative gastritis and esophagitis in children, with delayed gastric emptying as possible predisposing factor. Diagnosis is usually established by typical histological findings and can be confirmed by means of molecular detection techniques. Since infection may lead to life-threatening complications, it should be treated promptly with proton pump inhibitor and antibiotics. 


\section{ETHICS STATEMENT}

This study was carried out in accordance with the recommendations of the Ethical Commitee VU University medical center with written informed consent from all included subjects. The parents of these subjects gave written informed consent in accordance with the Declaration of Helsinki. The protocol was approved by the Ethical Commitee VU University medical center.

\section{REFERENCES}

1. Goodsir J. History of a case in which a fluid periodically ejected from the stomach contained vegetable organisms of an undescribed from. Edinb Med Surg J (1842) 57:430-43.

2. Edwards GT, Woodger NG, Barlow AM, Bell SJ, Harwood DG, Otter A, et al. Sarcina-like bacteria associated with bloat in young lambs and calves. Vet Rec (2008) 163:391-3. doi:10.1136/vr.163.13.391

3. Marshall TS. Abomasal ulceration and tympany of calves. Vet Clin North Am Food Anim Pract (2009) 25:209-20. doi:10.1016/j.cvfa.2008.10.010

4. Tuuminen T, Suomala P, Vuorinen S. Sarcina ventriculi in blood: the first documented report since 1872. BMC Infect Dis (2013) 13:169. doi:10.1186/1471-2334-13-169

5. Tolentino LF, Kallichanda N, Javier B, Yoshimori R, French SW. A case report of gastric perforation and peritonitis associated with opportunistic infection by Sarcina ventriculi. Lab Med (2003) 34:535-7. doi:10.1309/ CDFF04HE9FHDQPAN

6. Laass MW, Pargac N, Fischer R, Bernhardt H, Knoke M, Henker J. Emphysematous gastritis caused by Sarcina ventriculi. Gastrointest Endosc (2010) 72:1101-3. doi:10.1016/j.gie.2010.02.021

7. Lam-Himlin D, Tsiatis AC, Montgomery E, Pai RK, Brown A, Razavi M, et al. Sarcina organisms in the gastrointestinal tract: a clinicopathologic and molecular study. Am J Surg Pathol (2011) 35:1700-5. doi:10.1097/PAS.0b013e3182 2911 e6

8. Sauter JL, Nayar SK, Anders PD, D’Amico M, Butnor KJ, Wilcox RL. Co-existence of Sarcina organisms and Helicobacter pylori gastritis/duodenitis in pediatric siblings. J Clin Anat Pathol (JCAP) (2013) 1:103.

9. Ratuapli SK, Lam-Himlin DM, Heigh RI. Sarcina ventriculi of the stomach: a case report. World J Gastroenterol (2013) 19:2282-5. doi:10.3748/wjg.v19. i14.2282

10. Louis GB, Singh P, Vaiphei K. Sarcina infection. BMJ Case Rep (2014) 2014:bcr2013201185. doi:10.1136/bcr-2013-201185

11. Kumar M, Bhagat P, Bal A, Lal S. Co-infection of Sarcina and Giardia in a child. Oxf Med Case Reports (2014) 2014:118-9. doi:10.1093/omcr/omu046

12. Karakus E, Kirsaclioglu CT. Coincidence of celiac disease with Sarcina infection. Turk J Gastroenterol (2014) 25(Suppl 1):318. doi:10.5152/tjg. 2014.8028

13. Bhagat P, Gupta N, Kumar M, Radotra BD, Sinha SK. A rare association of Sarcina with gastric adenocarcinoma diagnosed on fine-needle aspiration. J Cytol (2015) 32:50-2. doi:10.4103/0970-9371.155238

\section{AUTHOR CONTRIBUTIONS}

TM is treating physician of described patients and wrote first draft of the manuscript. MW performed the endoscopies and critically assessed the manuscript. AM performed histological analyses and critically assessed the manuscript. $\mathrm{AB}$ performed microbiota analysis and critically assessed the manuscript.

14. Carrigan S, Grin A, Al-Haddad S, Iakovlev V, Streutker C, Moore T, et al. Emphysematous oesophagitis associated with Sarcina organisms in a patient receiving anti-inflammatory therapy. Histopathology (2015) 67:270-2. doi:10.1111/his.12599

15. Berry AC, Mann S, Nakshabendi R, Kanar O, Cruz D. Gastric Sarcina ventriculi: incidental or pathologic? Ann Gastroenterol (2015) 28:495.

16. Chougule A, Muthu V, Bal A, Rudramurthy SM, Dhooria S, Das A, et al. Pulmonary gangrene due to Rhizopus spp., Staphylococcus aureus, Klebsiella pneumoniae and probable Sarcina organisms. Mycopathologia (2015) 180:131-6. doi:10.1007/s11046-015-9904-3

17. Sopha SC, Manejwala A, Boutros CN. Sarcina, a new threat in the bariatric era. Hum Pathol (2015) 46:1405-7. doi:10.1016/j.humpath.2015.05.021

18. Medlicott SAC, Adams F. Sarcina ventricularis complicating a patient status post vertical banded gastroplasty: a case report. J Gastroenterol Hepatol Res (2015) 14:1481-4. doi:10.17554/j.issn.2224-3992.2015.04.498

19. Haroon Al Rasheed MR, Kim GJ, Senseng C. A rare case of Sarcina ventriculi of the stomach in an asymptomatic patient. Int J Surg Pathol (2015) 24(2): 142-5. doi:10.1177/1066896915610196

20. Ferrier D. The constant occurrence of Sarcina ventriculi (Goodsir) in the blood of man and the lower animals: with remarks on the nature of sarcinous vomiting. Br Med J (1872) 1(578):98-9. doi:10.1136/bmj.1.578.98

21. Gaspar BL. The significance of Sarcina in routine surgical pathology practice. APMIS (2016) 124(6):436-43. doi:10.1111/apm.12526

22. Canale-Parola E, Wolfe R. Studies on Sarcina ventriculi. I. Stock culture method. J Bacteriol (1960) 79:857-9.

23. Budding AE, Grasman ME, Lin F, Bogaards JA, Soeltan-Kaersenhout DJ, Vandenbroucke-Grauls CM, et al. IS-pro: high-throughput molecular fingerprinting of the intestinal microbiota. FASEB J (2010) 24(11):4556-64. doi:10.1096/fj.10-156190

Conflict of Interest Statement: The authors declare that the research was conducted in the absence of any commercial or financial relationships that could be construed as a potential conflict of interest.

Copyright (C) 2017 de Meij, van Wijk, Mookhoek and Budding. This is an open-access article distributed under the terms of the Creative Commons Attribution License (CC BY). The use, distribution or reproduction in other forums is permitted, provided the original author(s) or licensor are credited and that the original publication in this journal is cited, in accordance with accepted academic practice. No use, distribution or reproduction is permitted which does not comply with these terms. 